\title{
Brightness inhibition re size of surround'
}

\author{
LABORATORY OF PSYCHOPHYSICS, HARVARD UNIVERSITY
}

The amount by which the apparent brightness of a visual field is inhibited by a surrounding field depends on the area of the inhibiting field. Interocular brightness matches showed that, as the size of a surrounding annulus is increased from a thin ring, the degree of inhibition on the brightness of an inner disk increases rapidly at first and then more slowly as the effect approaches an asymptote. The increase of the inhibition with size of annulus can be expressed as an increase in the exponent of the power function that relates the apparent brightness of the disk to its physical luminance.

The degree to which one luminous field may mask or inhibit the apparent brightness of another luminous field (simultaneous brightness contrast) depends on several variables, such as the relative luminances and areas of the two fields (the subject of the present investigation), the geometric configuration formed by the fields, and their locations on the retina. (For reviews, see Brown \& Mueller, 1965; Diamond, 1960; Hurvich \& Jameson, 1966; and S. S. Stevens \& Diamond, 1965.)

In an effort to describe the visual masking that occurs in simultaneous contrast, a number of generalizations have been suggested, three of which may be summarized here.

(1) Simultaneous contrast seems to be effected mainly, if not solely, by a process of inhibition, so that the more luminous of two fields suppresses the brightness of the less luminous field, but the less luminous field leaves the brightness of the more luminous field unaltered (Diamond, 1953, 1955, 1962; S. S. Stevens \& Diamond, 1965).

(2) The amount by which the less luminous field is suppressed can be expressed as a change in the exponent of the power function that relates the apparent brightness $\psi$ to the luminance $\phi$ of the stimulating field. The power function may be written as $\psi=\mathrm{k} \phi \beta$ (S. S. Stevens, 1961, 1966). A single (uninhibited) field, viewed by the dark-adapted eye, yields an exponent $\beta$ equal to approximately 0.33 , but a field inhibited by simultaneous contrast may yield an exponent several times larger than 0.33 . The larger exponent applies only so long as the field is inhibited by a masking field, i.e., so long as its luminance is less than that of the masking field.

(3) The power function seems to hold for a variety of geometric relations between the inhibiting field and the inhibited field (Horeman, 1965; S. S. Stevens \& Diamond, 1965). No matter what the configuration, however, the value of $\beta$ for the inhibited field typi- cally increases with increasing luminance of the inhibiting field (S. S. Stevens, 1961)。S. S. Stevens (1966) has also compiled extensive evidence to show that the same kind of "power transformation" takes place under auditory masking, which suggests that the inhibition encountered in vision and in hearing may be mediated by similar mechanisms.

The relative size of $\beta$ can serve as a measure of how the degree of contrast varies with a given parameter of stimulation. For example, S. S. Stevens and Diamond (1965) showed that $\beta$ decreases systematically as the angle that separates the inhibited field from a small glare source is increased. In the present investigation, one of the main goals was to learn whether a change in $\beta$ could also serve as an index of inhibition when the parameter is the relative areas of the inhibiting and inhibited fields.

The configuration used was a disk (inhibited field) surrounded by an annulus (inhibiting field), which is the same configuration that is provided by the LummerBrodhun cube utilized in some kinds of photometers. (The advantage to photometry is the precision afforded by the high degree of inhibiting contrast produced by the disk-annulus configuration.) The increase in the exponent $\beta$ for the disk, as a function of the luminance of the inhibiting annulus, has already been investigated for this configuration (J. C. Stevens, 1957; S. S. Stevens, 1961). In the present experiment, the area of the annulus, rather than its luminance, was varied.

\section{Procedure}

Two identical configurations of disk and annulus were viewed separately by the two eyes, from a distance of $37 \mathrm{~cm}$. Each annulus had a luminance of $80 \mathrm{~dB}$ re $10^{-10} \mathrm{~L}(10 \mathrm{~mL})$. The two fields seen by the right eye had fixed areas throughout the study, as did the disk viewed by the left eye. The area of the annulus viewed by the left eye, however, was varied from time to time by means of circular masks placed directly in front of the annulus. Table 1 lists the dimensions of the various fields used.

Each configuration was produced by means of a pair of 100-W projector lamps, whose beams were diffused and viewed through a large Lummer-Brodhun cube. A description of these cubes and the accessory apparatus has been furnished by $S$. S. Stevens and Diamond (1965). The luminances of all four fields could be continuously adjusted over a large range by either the experimenter (E) or the observer (O).

Before an experimental session started, the $O$ dark-adapted for $5 \mathrm{~min}$. by wearing red goggles. He 
Table 1. Dimensions of the fields. The areas of the right and left targets, $T_{R}$ and $T_{L}$, were always the same $\left(0.25\right.$ in. $\left.{ }^{2}\right)$. The area of the right surround $\left(S_{R}\right)$ was fixed at 0.98 in. $^{2}$ and was equal to the largest value of the left surround $\left(S_{\mathbf{L}}\right)$.

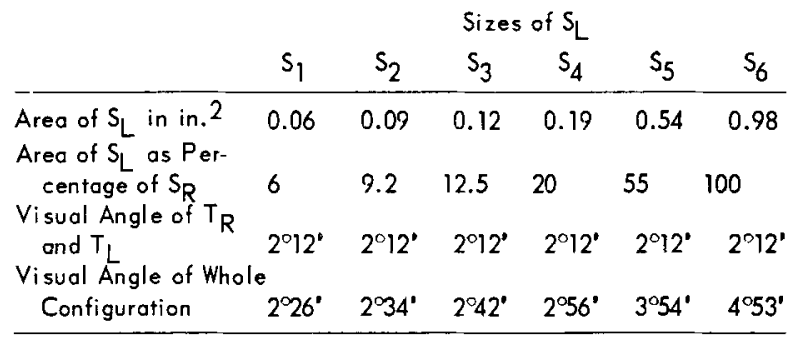

then continued to dark-adapt for another $2 \mathrm{~min}$. in the dark booth used for these experiments.

Each of 10 Os took part in two experiments, which differed only with respect to whether the $O$ adjusted the luminance of the right disk in the fixed surround or the left disk in the variable surround. In Experiment 1 , he adjusted the brightness of the right disk to match the brightness of the left disk. The E set the luminance of the left disk to the values 68,71 , 74 , and $77 \mathrm{~dB}$ re $10^{-10} \mathrm{~L}$, and the area of the left annulus to the six values given in Table 1 . Thus a total of 24 matches was called for in a session. Because of the high degree of contrast produced by the disk-annulus configuration, a reduction in the luminance of the disk from $80 \mathrm{~dB}$ to $68 \mathrm{~dB}$ causes the disk to plunge from moderately bright in appearance to very dark gray. In Experiment 2, the $O$ adjusted the left disk in order to match the brightness of the right disk, whose luminance was controlled by the $E$ (68, 71, 74, and $77 \mathrm{~dB}$ ). Otherwise the two experiments followed the same procedure. Half the Os served first in Experiment 1 and the other half served first in Experiment 2. At least a day separated the two sessions, and a typical session, including instructions and adaptation, lasted about 45 $\min$.

The $O$ was instructed to use the bracketing method of adjustment, that is to say, to begin by adjusting the brightness of the disk to appear clearly too bright and clearly too dim and then to "zero in" on the final setting. He was also instructed to keep his head and eyes aligned so as to see all four fields straight ahead. This was aided by resting the forehead against a partition that also functioned to permit each eye to see only the appropriate configuration.

When the two annuli have the same size, the two disks should appear equally bright when they have the same luminance. Actually, an adjustment bias usually causes the $O$ to make a small constant error, even under these physically equivalent conditions; When, however, the area of the left annulus is decreased, it loses some of its inhibitory effect on the left disk. As a consequence, the brightness of the left disk goes up, and it requires greater luminance in the right disk in order to match the left disk.
The method used here to measure inhibition might seem less straightforward than necessary. Why not dispense with the right annulus and allow the brightness of a single disk on the right to serve as an index of the brightness of the disk on the left? The answer is that under this condition it is far more difficult to effect a brightness match because a disk surrounded by an annulus and a single disk without a surround, display strikingly different modes of appearance. The surrounded disk resembles an illuminated surface that can be described as appearing "Iight gray," "medium gray," "black," etc., depending on its luminance relative to the surround. The disk without a surround does not appear to be a surface. The procedure of the present experiment was adopted because it helps to equalize the appearance of the disks.

Even so, there were numerous reports that a match seemed harder to make when the areas of the two annuli differed greatly. The reason may be simply that the decrease in inhibition caused by cutting down the size of one of the annuli may also diminish its surface-like character, thus bringing about a qualitative disparity between the two disks.

Table 2. For each of the four targets matched, the table shows the mean decibel settings and, in parentheses, the standard deviations for six sizes of the annulus $s$. Target levels are in decibels re $10^{-10}$ lambert. Slopes of the straight lines fitted by least squares and values of the Pearson correlation coefficient $r$ are also given.

\begin{tabular}{|c|c|c|c|c|c|c|}
\hline \multirow[t]{2}{*}{ Target Motched } & \multicolumn{6}{|c|}{$\begin{array}{c}\text { Experiment } 1 \\
\text { (Right target adjuste }\end{array}$} \\
\hline & $s_{1}$ & $\mathrm{~S}_{2}$ & $\mathrm{~S}_{3}$ & $\mathrm{~S}_{4}$ & $\mathrm{~S}_{5}$ & $S_{6}$ \\
\hline 77 & $\begin{array}{l}78.7 \\
(0.85)\end{array}$ & $\begin{array}{l}78.7 \\
(0.62)\end{array}$ & $\begin{array}{l}78.3 \\
(0.79)\end{array}$ & $\begin{array}{l}78.0 \\
(0.82)\end{array}$ & $\begin{array}{l}77.6 \\
(0.63)\end{array}$ & $\begin{array}{l}77.6 \\
(0.49)\end{array}$ \\
\hline \multirow[t]{2}{*}{74} & 76.8 & 76.3 & 75.6 & 75.4 & 74.3 & 74.2 \\
\hline & $(1.43)$ & $(1.27)$ & $(1.10)$ & $(0.99)$ & $(0.83)$ & $(0.96)$ \\
\hline 71 & $\begin{array}{l}75.0 \\
(1.44)\end{array}$ & $\begin{array}{l}74.0 \\
(1.46)\end{array}$ & $\begin{array}{l}73.8 \\
(1.20)\end{array}$ & $\begin{array}{l}73.4 \\
(1.08)\end{array}$ & $\begin{array}{l}72.0 \\
(0.99)\end{array}$ & $\begin{array}{l}72.0 \\
(1.43)\end{array}$ \\
\hline 68 & $\begin{array}{l}72.6 \\
(1.27)\end{array}$ & $\begin{array}{l}71.8 \\
(1.05)\end{array}$ & $\begin{array}{l}71.5 \\
(0.92)\end{array}$ & $\begin{array}{l}71.5 \\
(1.44)\end{array}$ & $\begin{array}{l}69.5 \\
(0.74)\end{array}$ & $\begin{array}{l}69.4 \\
(1.24)\end{array}$ \\
\hline Slope & 0.66 & 0.77 & 0.74 & 0.71 & 0.89 & 0.89 \\
\hline Pearson r & 0.997 & \multicolumn{2}{|c|}{$0.999+0.998$} & 0.998 & 0.997 & 70.996 \\
\hline \multicolumn{7}{|l|}{ Slope os Percentage } \\
\hline of Lorgest Slope & 74 & 86 & 83 & 80 & 100 & 100 \\
\hline \multirow[t]{2}{*}{ Target Matched } & \multicolumn{6}{|c|}{$\begin{array}{c}\text { Experiment } 2 \\
\text { eft target adjusted) }\end{array}$} \\
\hline & $s_{1}$ & $\mathrm{~S}_{2}$ & $\mathrm{~S}_{3}$ & $S_{4}$ & $\mathrm{~S}_{5}$ & $S_{6}$ \\
\hline 77 & $\begin{array}{l}74.9 \\
(1.57)\end{array}$ & $\begin{array}{l}75.9 \\
(1.06)\end{array}$ & $\begin{array}{l}76.4 \\
(0.91)\end{array}$ & $\begin{array}{l}76.6 \\
(0.49)\end{array}$ & $\begin{array}{l}77.1 \\
(0.51)\end{array}$ & $\begin{array}{l}77.4 \\
(0.25)\end{array}$ \\
\hline \multirow[t]{2}{*}{74} & 70.6 & 72.0 & 72.9 & 73.1 & 74.2 & 74.6 \\
\hline & $(1.17)$ & $(1.38)$ & $(0.86)$ & $(0.85)$ & $(0.64)$ & $(0.63)$ \\
\hline 71 & $\begin{array}{l}66.1 \\
(1.66)\end{array}$ & $\begin{array}{l}68.0 \\
(2.04)\end{array}$ & $\begin{array}{l}68.1 \\
(1.40)\end{array}$ & $\begin{array}{l}69.4 \\
(1.21)\end{array}$ & $\begin{array}{l}71.0 \\
(0.73)\end{array}$ & $\begin{array}{l}71.2 \\
(0.74)\end{array}$ \\
\hline 68 & $\begin{array}{l}60.1 \\
(2.50)\end{array}$ & $\begin{array}{l}62.4 \\
(2.75)\end{array}$ & $\begin{array}{l}65.5 \\
(1.48)\end{array}$ & $\begin{array}{l}65.5 \\
(0.67)\end{array}$ & $\begin{array}{l}67.5 \\
(0.63)\end{array}$ & $\begin{array}{l}68.3 \\
(1.02)\end{array}$ \\
\hline Slope & 0.61 & 0.67 & 0.80 & 0.81 & 0.94 & 0.98 \\
\hline \multirow{2}{*}{$\begin{array}{l}\text { Pearson } r \\
\text { Slope as Percentage } \\
\text { of Laraest Slope }\end{array}$} & 0.997 & 0.997 & 0.995 & $0.999+$ & +0.999 & 90.999 \\
\hline & 62 & 68 & 82 & 83 & 96 & 100 \\
\hline
\end{tabular}




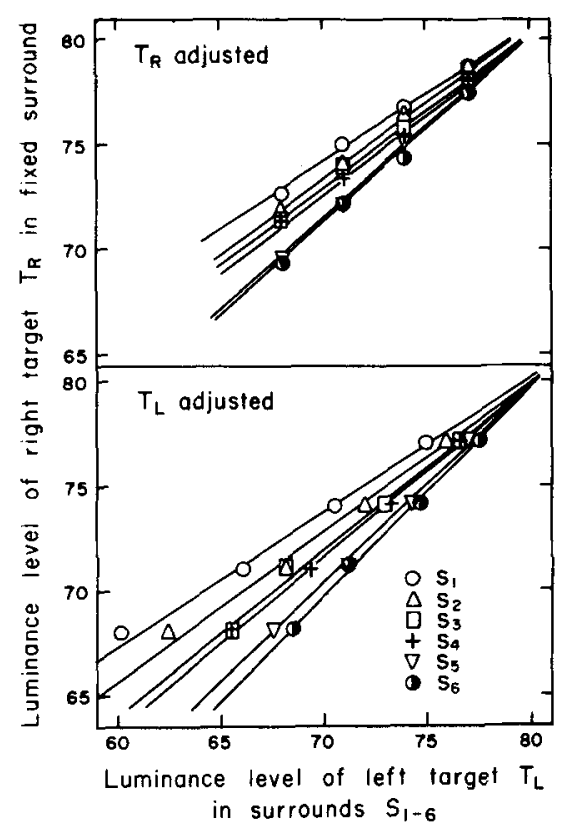

Fig. 1. Showing how the annulus size $S$ affects the brightness exponent. The left target disk $T_{L}$ in a variable annulus and the right disk $T_{R}$ in a fixed annulus were made to appear equal in brightness by the observer. For the upper graph the luminance of $T_{R}$ was adjusted; for the lower graph the luminance of $T_{L}$ was adjusted. Values of $S$ are given in Table 1 .

\section{Results}

The variability of the settings (see Table 2) tended to be higher when the areas of the two annuli were very different. This relation was clear-cut when the left target, in the variable annulus, was adjusted (Experiment 2).

The mean decibel settings are listed in Table 2 and plotted in Fig. 1 (where area is the parameter) and in Fig. 2 (where luminance is the parameter). Figure 1 demonstrates that the change in the area of the annulus causes a "power transformation" of the brightness function, whereas Fig. 2 shows more directly how the degree of suppression depends on the size of the annulus.

The points plotted in the decibel (logarithmic) coordinates of Fig. 1 were fitted with straight lines by the method of least squares. These lines have the equation:

$$
\log \phi_{\mathrm{R}}=\gamma \log \phi_{\mathrm{L}}+\log \mathrm{C}
$$

where $\phi_{\mathrm{L}}$ is the luminance of the left target, and $\phi \mathbf{R}$ is the luminance of the right target. The slope $y$ decreases as the area of the left annulus is decreased. Equation (1) can also be written in its non-logarithmic form:

$$
\phi_{\mathrm{R}}=\mathrm{C} \phi_{\mathrm{L}} \gamma
$$

The slopes (or exponents) of the fitted functions are given in Table 2, together with the Pearson coefficient of correlation for each function. The generally satisfactory fit of a straight line supports the hypothesis that the effect of changing the area of the inhibiting field can be specified by a change in the exponent of Equation (2).

It also follows that if the apparent brightness $\psi$ of a disk surrounded by an annulus of any given area grows as a power function of the disk luminance $\phi$ according to $\psi=\mathrm{k} \phi \beta$, then the disk brightness must grow as a power function of luminance regardless of the area of the annulus. In other words, the power function that relates the sensation to the stimulus magnitude stays invariant in form, but the size of the exponent depends on the relative areas of disk and surround. As the ratio of the annulus area to the disk area diminishes, the value of $\beta$ also diminishes.

Ideally, all the lines in Fig. 1 should pass through the point defined by $80 \mathrm{~dB}$ on both coordinates, the fixed luminance level of the two annuli. In fact, the projections of the lines in Fig. 1 tend to miss the predicted equality mark by a small amount-on the average by about $0.35 \mathrm{~dB}$. With the right disk adjusted, the lines project above the predicted point, but with the left disk adjusted, the lines project below the predicted point.

An error of adjustment also shows up in the slopes of the two equal brightness functions determined under the condition that the right and left annuli have the same area. Ideally these slopes should equal unity, but slightly smaller values $(0.98$ and 0.89$)$

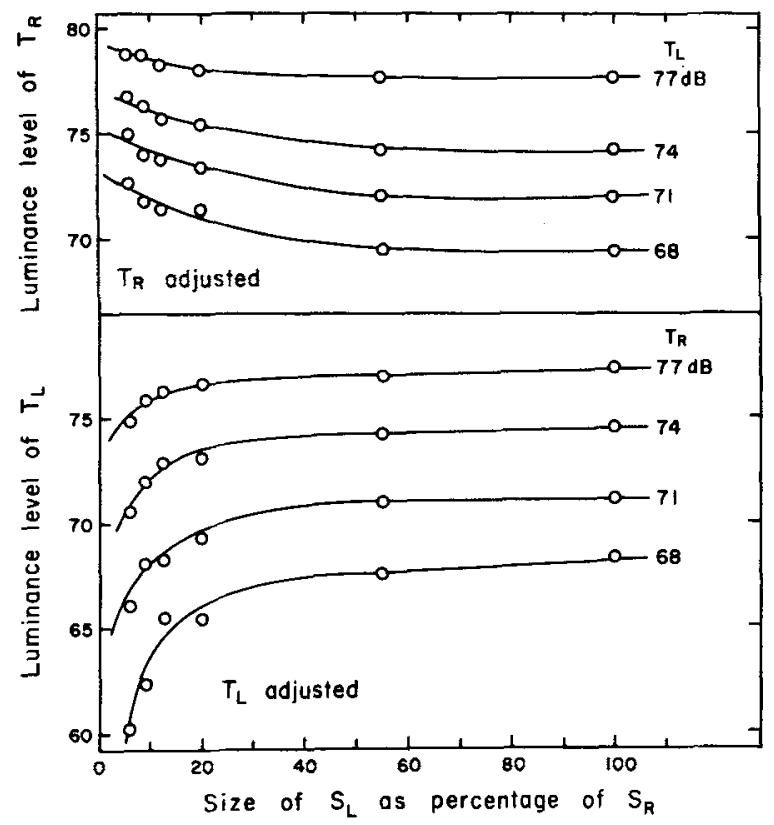

Fig. 2. Showing the disk luminance (ordinate) that appeared to match a given disk luminance (parameter) as a function of the relative area (abscissa) of the annulus surrounding the target $T_{L}$ seen by the left eye. 
were obtained. This type of "regression bias" is to be expected, for it pervades the domain of psychophysical measurement (S. S. Stevens \& Greenbaum, 1966).

Table 1 contains not only the absolute values of the fitted slopes, but also the same values expressed as percentages of the largest slopes obtained. Thus, the smallest annulus produced a disk exponent that was $74 \%$ (Experiment 1) or $62 \%$ (Experiment 2) as large as the disk exponent corresponding to the largest annulus. This power transformation represents a sizeable change in the psychophysical function. By way of comparison, it may be noted that light adaptation also causes a change in the exponent of the power function for the brightness of a single (uninhibited) target (Onley, 1961; Stevens \& Stevens, 1963; van den Brink, 1962). For the dark-adapted eye the exponent $(0.33)$ is about $70 \%$ as large as the exponent $(0.45)$ for the eye adapted to $1 \mathrm{~L}$.

Figure 2 shows how the degree of inhibition increases as the size of the annulus is increased. The most prominent feature of these curves is the rapid initial increase of inhibition as the size of the annulus is increased from a tiny ring about $7 \mathrm{~min}$. wide, the smallest annulus used. The inhibition increases rapidly until the annulus is about half a degree wide, but thereafter further increase in the size of the annulus seems to add little to the degree of inhibition. An annulus larger than the largest field used (1 degree, 2 min. wide) would presumably contribute little or no additional increment of inhibition. No matter what index of size is plotted-the area of the left annulus relative to the area of the right annulus as in Fig. 2, the area of the left annulus relative to the area of the left target, or the relative angular width of the annulus-all these indices show that the primary inhibitory effect is exerted by a relatively small portion of the annulus adjacent to the inhibited disk. The degree of inhibition also declines rather sharply with increased retinal separation between the inhibited and inhibiting fields, although with a small glare source and a small target, the inhibiting effect was measurable up to separations of about 4 degrees (S. S. Stevens \& Diamond, 1965). Thus, in terms of retinal angle, the effect of adding area to an annulus approaches its asymptote more rapidly than the effect of moving a glare source away from a target.

\section{References}

Brown, J. L., \& Mueller, C. G. Brightness discrimination and brightness contrast. In C. H. Graham (Ed.), Vision and visual perception. New York: Wiley, 1965. Pp. 208-250.

Diamond, A. L. Foveal simultaneous brightness contrast as a function of inducing- and test-field luminances. J. exp. Psychol. $1953,45,304-314$.

Diamond, A. L. Foveal simultaneous contrast as a function of of inducing-field area. J. exp. Psychol., 1955, 50, 144-152.

Diamond, A. L. A theory of depression and enhancement in the brightness response. Psychol. Rev., 1960, 67, 168-199.

Diamond, A. L. Simultaneous contrast as a function of test-field area, J. exp. Psychol., 1962, 64, 336-345.

Hurvich, L. M., \& Jameson, D. The perception of brightness and darkness. Boston: Allyn and Bacon, 1966.

Horeman, $\mathbf{H}$. $\mathbf{W}$. Relations between brightness and luminance under induction. Vision Res., 1965, 5, 331-340.

Onley, J. Light adaptation and the brightness of brief foveal stimuli. J. Opt. Soc. Amer., 1961, 51, 667-673.

Stevens, J. C. A comparison of ratio scales for the loudness of white noise and the brightness of white light. Ph.D. dissertation, Harvard University, 1957

Stevens, J. C., \& Stevens, S. S. Brightness function: Effects of adaptation. J. Opt. Soc. Amer., 1963, 53, 375-385.

Stevens, S. S. To honor Fechner and repeal his law. Science, 1961 $133,80-86$.

Stevens, S. S. Power-group transformations under glare, masking, and recruitment. J. Acoust. Soc. Amer., 1966, 39, 725-735.

Stevens, S. S., \& Diamond, A. L. Effect of glare angle on the brightness function for a small target. Vision Res., 1965, 5, 649-659.

Stevens, S. S., \& Greenbaum, H. Regression effect in psychophysical judgment. Percept. \& Psychophys., 1966, 1, 439-446.

van den Brink, G. Subjective brightness during dark-adaptation. Vision Res., 1962, 2, 495-502.

\section{Notes}

1. Research supported in part by National Institutes of Health Grant NB-02974 and in part by National Science Foundation Grant GB-3211 (Laboratory of Psychophysics Report PPR-333).

2. Now at John B. Pierce Foundation Laboratory, and Department of Psychology, Yale University, New Haven, Connecticut. 